\title{
OBTENÇÃO DE RESULTADOS QUANTITATIVOS PARA CONCENTRAÇÃO DE CHUMBO EM SOLO COM EQUIPAMENTO XRF PORTÁTIL
}

\author{
DETERMINING QUANTITATIVE LEAD CONCENTRATION IN SOIL WITH PORTABLE XRF \\ EQUIPMENT
}

\author{
Samuel Gonçalves SOUZA CRUZ ${ }^{1}$, Ana Elisa SILVA DE ABREU ${ }^{2}$ \\ ${ }^{1}$ Curso de Especialização em Investigação do Subsolo: Geotecnia e Meio Ambiente - Instituto de Pesquisas Tecnológicas, SP. \\ E-mail: samuel.gscruz@gmail.com \\ ${ }^{2}$ Departamento de Geologia e Recursos Naturais, Instituto de Geociências, Unicamp. Rua Carlos Gomes, 250 - Cidade Universitária, \\ Campinas - SP, SP. E-mail: anaelisa@ige.unicamp.br

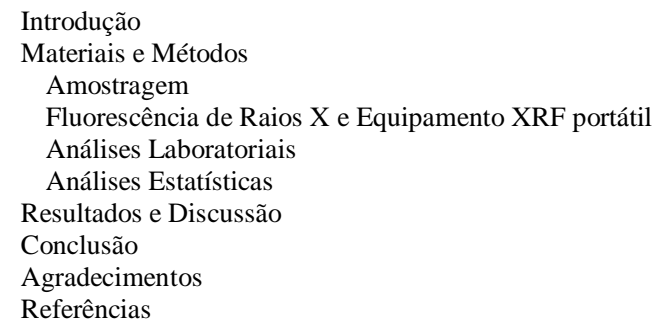

RESUMO - O chumbo está entre as substâncias químicas consideradas contaminantes por diversos órgãos ambientais nacionais e internacionais. Para a detecção deste elemento em solo são realizados testes com aparelhos portáteis de fluorescência de raios x (XRFP), pois estes equipamentos apresentam inúmeras vantagens, como sua portabilidade, facilidade de uso, baixo custo, alta velocidade, alta precisão e detecção não destrutiva. Neste trabalho foi verificada a possibilidade da utilização deste equipamento para a realização de levantamentos quantitativos de $\mathrm{Pb}$ em amostras de solo superficial coletadas no interior e adjacências de uma área sob investigação ambiental. Tal verificação foi feita por meio de testes estatísticos entre os resultados obtidos pelo XRF-P e por ensaios laboratoriais normatizados realizados em amostras pareadas. Com o tratamento estatístico aplicado aos dados, foi possível desenvolver uma equação de regressão que possibilita a estimativa de valores confiáveis de concentrações de $\mathrm{Pb}$ no solo, a partir dos resultados obtidos pelo XRF-P para a área estudada. Os resultados obtidos com XRF-P neste caso são classificados como definitivos segundo USEPA (1988) e quantitativos segundo Shuttleworth et al. (2014).

Palavras-chave: Contaminação, Solo Superficial, XRF-P, Teste-t pareado.

\begin{abstract}
Lead is an element considered a contaminant by various national and international environmental agencies. Tests with portable x-ray fluorescence equipment (XRF-P) are performed in soil for the detection of this element, since these devices have many advantages, such as portability, ease of use, low cost, high speed, high accuracy and non destructive detection. The possibility of using this equipment to perform quantitative surveys of $\mathrm{Pb}$ in surface soils in the interior and neighborhoods of an area under environmental investigation was verified in this study. Such verification was made through linear regressions and statistical tests between the results obtained by XRF-P and standardized laboratory tests performed on paired samples. With the statistical treatment applied to the data, it was possible to develop a regression equation that allows the calculation of reliable quantitative $\mathrm{Pb}$ concentrations in the soil from the results obtained by the XRF-P for the studied area. The data obtained by XRF-P was classified as definitive according to USEPA (1988) and quantitative according to Shuttleworth et al. (2014).
\end{abstract}

Keywords: Contamination, Topsoil, XRF-P, Paired t-test.

\section{INTRODUÇÃO}

Elementos-traço são elementos que ocorrem em concentrações inferiores a $0,1 \%$, em materiais naturais e incluem metais traço, metais pesados, semi-metais, micronutrientes, entre outros (Sparks, 1995). Determinados elementostraço são essenciais aos seres-vivos, porém em algumas circunstâncias, podem gerar impactos negativos em ecossistemas terrestres e aquáticos (Guilherme et al., 2005). Seres vivos, quando expostos a concentrações específicas de elementos-traço, podem apresentar sérias patologias, como por exemplo a não germinação de sementes ou subdesenvolvimento de plantas (Aydinalp \& Marinova, 2000). Dentre os malefícios está incluída a toxidade aos seres humanos.

O chumbo, amplamente distribuído como chumbo metálico, compostos inorgânicos e compostos organometálicos, apresenta vários efeitos tóxicos ao organismo humano, incluindo a inibição da síntese de hemoglobina, afetando também negativamente o sistema nervoso central e periférico, e os rins (Manahan, 2000). Por este motivo o chumbo está entre as substâncias 
químicas consideradas contaminantes dos solos, águas superficiais e subterrâneas, por diversos órgãos ambientais nacionais e internacionais, como a Companhia Ambiental do Estado de São Paulo (CETESB) e United States Environmental Protection Agency (USEPA). Estes órgãos definem valores de concentração padrões das substâncias nocivas à saúde humana, cenários de exposição para estas substâncias, atividades que são potenciais contaminantes, e exigem legalmente investigações ambientais de áreas potencialmente contaminadas, e quando confirmada a contaminação e risco a saúde humana, recuperação e remediação destas áreas.

Os metais pesados e semi-metais traços presentes nos solos podem ser derivados do material parental do solo (fonte litogênica) e existem muitas fontes antropogênicas de contaminação de metais pesados que afetam tanto solos agrícolas quanto solos urbanos (Alloway, 2013). Dentre as fontes antropogênicas estão a queima de combustíveis fósseis, atividades industriais e minerais específicas.

Operações de mineração e de metalurgia, quando localizadas no interior de áreas urbanas ou em suas adjacências podem configurar uma importante fonte de emissão de metais traço na atmosfera. Após a emissão os metais-traço tendem a aderir a partículas finas em suspensão, estas são transportadas pelo vento e depositadas no solo e na água (Wong et al., 2006).

Para a investigação de áreas potencialmente contaminadas, são empregados diversos métodos de amostragem e análise de água e solo, regulamentados pelos órgãos ambientais como os acima citados, e normativas nacionais e internacionais. Em áreas com suspeita de contaminação de solo por metais, são empregados métodos de análises laboratoriais já tradicionais como fluorescência de raios $\mathrm{X}$ (XRF), e espectrometria de absorção atômica por chama (FLAA) (Davidson, 2013). Para análise de elementos-traços no solo tem sido utilizada análise por meio de aparelhos de fluorescência de raios $\mathrm{x}$ portáteis (XRF-P). Trata-se de um método de detecção com inúmeras vantagens, incluindo sua portabilidade, facilidade de uso, baixo custo, alta velocidade, alta precisão e detecção não destrutiva (DiScenza et al, 2014; Hu et al., 2017).

No geral, os resultados obtidos com equipamentos XRF-P são indicados para trabalhos de varredura nas fases iniciais de investigação de áreas contaminadas (CETESB, 1999), sendo considerados geralmente como resultados qualitativos. Entretanto, Kilbride et al. (2006), Shuttleworth et al. (2014) e Rouillon \& Taylor (2016) têm demonstrado que estes equipamentos podem fornecer resultados quantitativos.

De acordo com USEPA (1998), os dados obtidos pelo aparelho de XRF-P possuem caráter qualitativo, quando indicam a presença ou não de determinado elemento na amostra, não havendo precisão ou confiabilidade na quantidade e concentração do elemento em questão. Eles são considerados quantitativos, quando além da sinalização dos elementos, o método indica valores confiáveis de quantidades ou concentrações destes elementos. Quando se pode atestar que os valores obtidos indicam valores muito próximos do real, ou seja, o equipamento apresenta precisão e acurácia, diz-se que se trata de um método de investigação com resultados definitivos. USEPA(1998) propõe um critério para avaliação da qualidade dos dados obtidos, conforme exposto na Tabela 1. Por este critério são definidos três níveis de qualidade das regressões entre os dados e os parâmetros que definem cada um destes. Nesta proposta avaliase a acurácia por meio do coeficiente de determinação $\left(\mathrm{R}^{2}\right)$ da regressão linear dos resultados obtidos com equipamento XRF-P, com os resultados obtidos em laboratório. A precisão é avaliada por meio do desvio padrão relativo (RSD).

Tabela 1 - Critério de análise da qualidade da regressão linear dos dados (Modificado de USEPA, 1998).

\begin{tabular}{c|c}
\hline Nível de qualidade do dado & Parâmetros Estatísticos Exigidos \\
\hline \multirow{2}{*}{ Definitivo } & $\mathrm{R}^{2 *}=0,85$ a 1 \\
\cline { 2 - 2 } Quantitativo & $\mathrm{RSD}^{* *}<10 \%$ \\
\cline { 2 - 2 } & $\mathbf{R}^{2}=\mathbf{0 , 7}$ a 1 \\
\hline \multirow{2}{*}{ Qualitativo } & $\mathrm{RSD}<20 \%$ \\
\cline { 2 - 2 } $\mathrm{R}^{2}<0,7$ \\
\hline $\mathrm{R}^{2}$ : Coeficiente de determinação. **RSD: Desvio padrão relativo
\end{tabular}


O modelo de regressão linear é representado pela Equação 1. Ele minimiza o quadrado da diferença entre a variável dependente e a linha de regressão (Kilbride et al., 2006).

$$
\mathrm{Y}=\mathrm{mx}+\mathrm{c} \quad \text { (Equação 1) }
$$

Onde, Y são valores relativos aos ensaios laboratoriais (variável dependente), $\mathrm{x}$ a concentração de $\mathrm{Pb}$ obtido pelo equipamento de XRF-P (variável independente), c a intersecção da reta no eixo y e $m$ é a inclinação da reta de regressão linear.

$\mathrm{O}$ coeficiente de determinação $\left(\mathrm{R}^{2}\right)$ é um dos meios para se avaliar se duas variáveis estão correlacionadas. Ele mede quanto da variabilidade do modelo é explicada pela correlação linear e deveria idealmente ser igual a 1 , isso indicaria um erro zero.

O RSD é um parâmetro normalmente utilizado como medida da precisão de um determinado equipamento ou método analítico. A precisão do método é monitorada a partir da análise de uma amostra com concentrações baixas, moderadas ou altas dos analitos de interesse. USEPA (2007) recomenda que, para a obtenção deste parâmetro, deve ser feita uma medida de precisão por dia e cada amostra deve ser analisada repetidamente sete vezes. Importante que seja feita a obtenção de medidas de precisão para amostras com diferentes intervalos de concentração, pois desta forma é possível avaliar o efeito da concentração na precisão dos métodos de análise.

A partir da média dos resultados obtidos nas análises, calcula-se o desvio padrão relativo (RSD). Consideram-se os dados de XRF-P precisos quando o RSD for inferior a $20 \%$. Para o cálculo do RSD é utilizada a Equação 2:

$$
\mathrm{RSD}=(\mathrm{SD} / \text { Concentração Média }) \times 100 \quad \text { (Equação 2) }
$$

Onde, RSD é o desvio padrão relativo para a medida de precisão para o analito, SD é o desvio padrão da concentração para o analito $(\mathrm{mg} / \mathrm{kg})$ e "Concentração Média" constitui a concentração média para o analito $(\mathrm{mg} / \mathrm{kg})$.

Alternativamente aos critérios de USEPA (1998), Shuttleworth et al. (2014) propuseram um esquema ainda mais rigoroso para a classificação de regressões obtidas a partir de dados instrumentais. Este esquema está sintetizado na Tabela 2 e considera, além do $\mathrm{R}^{2} \mathrm{e}$ do RSD, o uso de testes estatísticos para verificação da similaridade entre os resultados do padrão e os resultados obtidos pela regressão. Neste esquema o $\mathrm{R}^{2}$ é uma medida da força da associação linear entre os dois métodos de análise. O RSD é uma medida da precisão e o teste de hipótese ao nível de confiança de 5\%, tendo como hipótese nula a regressão linear com c igual a 0 e m igual a 1 é a medida da acurácia.

Tabela 2 - Critério de análise do nível de qualidade da regressão linear entre várias análises proposto por Shuttleworth

\begin{tabular}{|c|c|}
\hline $\begin{array}{l}\text { Nível de qualidade } \\
\text { do dado }\end{array}$ & Parâmetros Estatísticos Exigidos \\
\hline \multirow{3}{*}{ Definitivo } & $\mathrm{R}^{2}=0,85$ a1 \\
\hline & $\mathrm{RSD}<10 \%$ \\
\hline & $\begin{array}{l}\text { Os testes estatísticos devem indicar ao nível de confiança de } 5 \% \text { que os valores estimados } \\
\text { com a regressão } \mathrm{y}=\mathrm{mx}+\mathrm{c}, \mathrm{com} \mathrm{m}=1 \mathrm{e} \mathrm{c}=0 \text { são idênticos àqueles obtidos com o XRF-P. }\end{array}$ \\
\hline \multirow[b]{3}{*}{ Quantitativo } & $\mathrm{R}^{2}=0,7 \mathrm{a} 1$ \\
\hline & RSD $<20 \%$ \\
\hline & $\begin{array}{l}\text { Os testes estatísticos indicam ao nível de confiança de } 5 \% \text {, que os valores estimados com a } \\
\text { regressão } \mathrm{y}=\mathrm{mx}+\mathrm{c}, \text { com } \mathrm{m}=1 \mathrm{e} \mathrm{c}=0 \text { não são idênticos àqueles obtidos com o XRF-P. } \mathrm{y}= \\
\mathrm{mx}+\mathrm{c} \text { é aceita }\end{array}$ \\
\hline \multirow{2}{*}{ Qualitativo } & $\mathrm{R}^{2}<0,7$ \\
\hline & $\mathrm{RSD}<20 \%$ \\
\hline
\end{tabular}
et al. (2014).

O critério proposto por Shuttleworth et al. (2014) exige ainda que se verifique a distribuição dos resíduos da regressão linear, para que se possa discernir sobre a validade da mesma. A distribuição dos resíduos de uma regressão não deve exibir padrão que possa permitir a inferência sobre o resíduo de valores adjacentes entre si. Caso seja identificada alguma tendência os dados devem passar por uma transformação matemática ou deve-se estudar a conveniência de 
remover valores anômalos.

O objetivo deste trabalho foi o de verificar a eficácia do método de análise de $\mathrm{Pb}$ por meio de equipamento de XRF-P em amostras de solo superficial coletadas no interior e adjacências de uma antiga planta industrial metalúrgica, comparando e correlacionando seus resultados com resultados analíticos laboratoriais, e utilizar ferramentas estatísticas para avaliar se os resultados fornecidos por este equipamento podem ser considerados quantitativos e propor um método de tratamento destes dados, de forma a se obter indiretamente as concentrações de $\mathrm{Pb}$ no solo.

\section{MATERIAIS E MÉTODOS}

Os dados analisados no presente artigo foram obtidos em investigações ambientais executadas em uma unidade industrial metalúrgica no Estado de São Paulo, cujo período das atividades se deu entre os anos de 1974 e 1996. Nesta unidade era produzido óxido de chumbo, tendo como principais matérias primas no processo industrial o chumbo metálico, silicatos e óleo diesel.

Nas investigações ambientais realizadas nos anos de 2011 e 2012, seguindo padrões e normativas da época, foram analisados o solo superficial e a água subterrânea. Este trabalho limita-se aos dados obtidos em amostras de solo superficial, pois sobre estas concentram-se as investigações realizadas com equipamento XRF-P.

\section{Amostragem}

Os metais analisados nos testes via XRF-P foram antimônio, arsênio, cádmio, chumbo, cobre, cromo, níquel e zinco. Nos ensaios laboratoriais foram determinadas apenas as concentrações de chumbo.

As amostras de solo foram coletadas em 705 pontos de sondagem georreferenciados, locados no centro de uma malha de quadrados com 10 metros de lado, que compreende a área da antiga planta industrial e suas adjacências (Figura 1). Em cada ponto foram coletadas e separadas amostras através do método direct push, em três faixas de profundidades, entre 0,0 a $0,1 \mathrm{~m}, 0,1$ a $0,2 \mathrm{~m}$ e 0,2 a $0,3 \mathrm{~m}$, totalizando 2115 amostras.

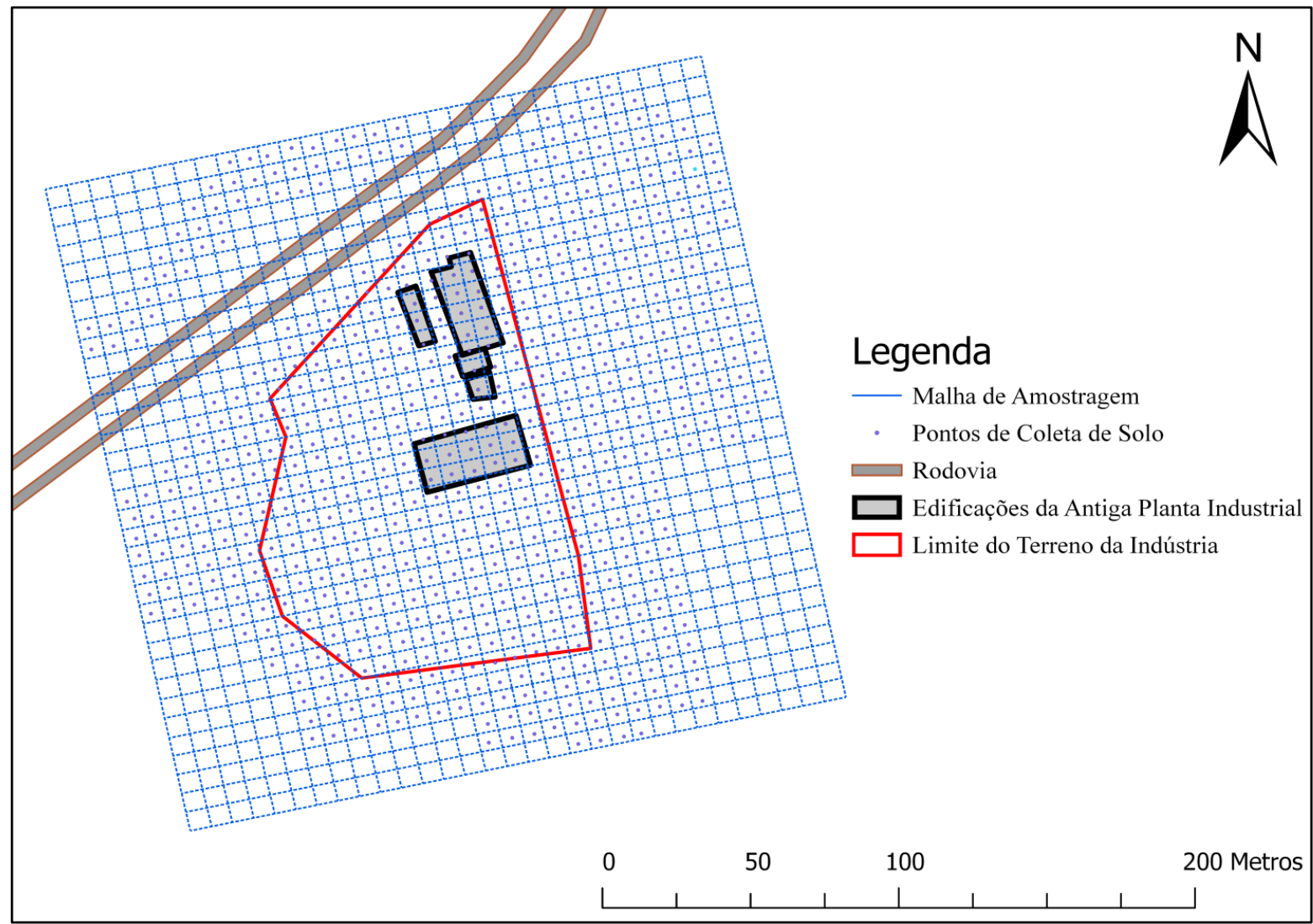

Figura 1 - Malha de Amostragem de 10 x 10 Metros e Localização dos 705 Pontos de Amostragem de Solo.

O método direct push consiste na cravação de um amostrador especial no solo utilizando-se para isso força hidráulica ou mecânica. O amostrador empregado neste estudo é revestido com um tubo de PEAD (Polietileno de Alta Densidade) com diâmetro de 2". A cravação do amostrador se deu em pontos seguindo uma malha pré-estabelecida, também indicada na 
figura 1, sendo que em pontos de amostragem onde havia piso de concreto, o mesmo foi rompido e coletadas amostras logo abaixo do piso.

Seguindo padrões da técnica de amostragem ISM (Incremental Sampling Methodology) e a norma ABNT NBR 10007/2004, cada uma das amostras foi desagregada em bandeja apropriada, em seguida homogeneizada, quarteada e peneirada na granulometria areia fina $(<0,25 \mathrm{~mm})$. Após este processo de tratamento das amostras, o volume das mesmas foi reduzido em cerca de $75 \%$, selecionando frações mais finas de solo, em seguida as amostras foram secas, evitando-se assim qualquer resultado analítico não satisfatório pelo excesso de umidade. Finalmente as alíquotas de solo assim preparado foram armazenadas em sacos plásticos de PEAD, livres de qualquer contaminação e em seguida submetidas a testes com o equipamento de XRF-P.

Amostras analisadas pelo instrumento XRF-P que apresentaram concentrações de chumbo acima do Limite de Detecção (11 mg/kg) foram selecionadas e enviadas para análises laboratoriais., totalizando 75 amostras para as quais se dispõe dos resultados para concentração de $\mathrm{Pb}$ determinadas tanto com equipamento portátil, quanto em laboratório. As demais amostras foram devidamente descartadas.

A análise dos resultados, tanto das análises laboratoriais quanto dos ensaios via XRF-P, foi realizada considerando-se os valores de intervenção da CETESB, para concentrações de substâncias contaminantes no solo. Valor de intervenção é a concentração de determinado contaminante na matriz analisada (solo ou água subterrânea) acima da qual existe risco à saúde humana, considerandose o cenário específico de exposição da área investigada. Neste trabalho foi considerado cenário agrícola, pois, apesar de ser uma antiga unidade industrial, hoje está desativada e sua vizinhança é composta por propriedades agrícolas e bairros residenciais, se tratando de contaminação presente no solo em ambiente agrícola, os valores de intervenção são os mais restritivos. À época da coleta dos dados estudados nesse artigo o valor de intervenção para chumbo em solo em cenário agrícola era de $180 \mathrm{mg} / \mathrm{kg}$ (CETESB, 2005). Atualmente o valor de intervenção é de $150 \mathrm{mg} / \mathrm{kg}$ (CETESB, 2016).

\section{Fluorescência de Raios X e Equipamento XRF Portátil}

Nos testes produzidos por meio de aparelhos XRF-P são utilizadas fontes de radioisótopos seladas em tubos de raios x. Estas fontes são responsáveis pela irradiação de raios x em amostras de solo ou sedimento. Quando uma amostra recebe a radiação, os raios $\mathrm{x}$ de origem podem sofrer dispersão ou absorção por átomos presentes na atmosfera. Havendo absorção define-se o processo como efeito fotoelétrico (USEPA, 2007).

À medida que um átomo absorve raios $\mathrm{x}$ da fonte emissora, os elétrons de níveis mais profundos do átomo são desalojados, produzindo vagas nestes níveis, que são preenchidas por elétrons de níveis mais externos, gerando um efeito em cascata. $\mathrm{O}$ rearranjo em cascata dos elétrons, a partir de níveis mais externos para níveis mais internos, produz uma emissão de energia. Quando há, através deste processo, a emissão de raios $\mathrm{x}$, cria-se o fenômeno conhecido como fluorescência de raios $\mathrm{x}$. A fluorescência de raios x produzida pelos átomos das amostras de solo, após o estímulo do aparelho XRF-P, é captada pelo mesmo, gerando um espectro de emissão no qual podem ser identificados quais elementos estão presentes na amostra e o teor de cada um deles.

Nesta investigação foi utilizado o aparelho XRF Analyzer Niton XL2 Gold D para os ensaios de campo e as amostras foram submetidas a apenas uma determinação cada. Este modelo de XRF-P, fabricado pela Thermo Scientific, possui um espectro de detecção de 25 elementos, compreendendo elementos entre o magnésio $(\mathrm{Mg})$ e o urânio (U) na tabela periódica e é equipado com tubo com ânodo de prata cuja tensão máxima é $45 \mathrm{kV}$ e a corrente máxima produzida é de $100 \mathrm{uA}$.

Este equipamento apresenta certificado de calibração de fábrica, além de possuir uma função de auto-calibração, que é realizada previamente a uma nova campanha de investigação de campo ou uma vez a cada semana, quando se fizer necessário. Após a auto-calibração é necessário realizar uma análise normal de uma amostra padrão que acompanha o equipamento e que detém certificação do National Institute of Standards and Technology (NIST).

Para a realização das leituras as amostras foram colocadas em uma campânula de chumbo, de tal forma que o operador do equipamento estivesse protegido contra as radiações emitidas pelo equipamento e cada leitura teve a duração de apenas um minuto.

\section{Análises Laboratoriais}

As análises laboratoriais seguiram rigorosamente as especificações e normativas para Método 3050B e 6010B da USEPA. O Método 3050B 
refere-se aos procedimentos para tratamento de amostras de solos, lamas e sedimentos por meio de digestão ácida, para a determinação da concentração de metais através de diferentes métodos analíticos laboratoriais, incluindo a espectrometria de emissão atômica por plasma acoplado indutivamente (ICP-AES). Este procedimento analítico é regulamentado pelo Método 6010B. Os limites de detecção e quantificação para chumbo do equipamento de ICP-AES, utilizado nos ensaios laboratoriais deste estudo, são respectivamente 1 $\mathrm{mg} / \mathrm{kg}$ e $4 \mathrm{mg} / \mathrm{kg}$.

O método analítico ICP-AES é utilizado para determinações multielementais através de sistemas ópticos sequenciais ou simultâneos e visão axial ou radial do plasma. $\mathrm{O}$ instrumento mede os espectros de emissão característicos dos elementos por espectrometria óptica. As amostras são nebulizadas e o aerossol resultante é transportado para uma chama de plasma. O plasma acoplado por radiofrequência produz espectros de emissão específicos de cada elemento, estes espectros são dispersos em uma grade espectométrica e as intensidades das linhas de emissão são monitoradas por meio de dispositivos fotossensíveis (USEPA, 1996).

\section{Análises Estastísticas}

Para a cálculo do modelo de regressão linear entre os dados obtidos com o equipamento XRF-P e aqueles obtidos por análises tradicionais em laboratório foi utilizado o software excell.

$\mathrm{O}$ teste t-pareado foi utilizado como ferramenta estatística para teste de hipótese e para verificação da similaridade entre grupos amostrais, considerando-se nível de significância de 5\%. Para tal verificação foi utilizado o software online "SCICALC".

O fluxograma apresentado na figura 2 resume o andamento das verificações quanto à qualidade das correlações realizadas neste estudo.

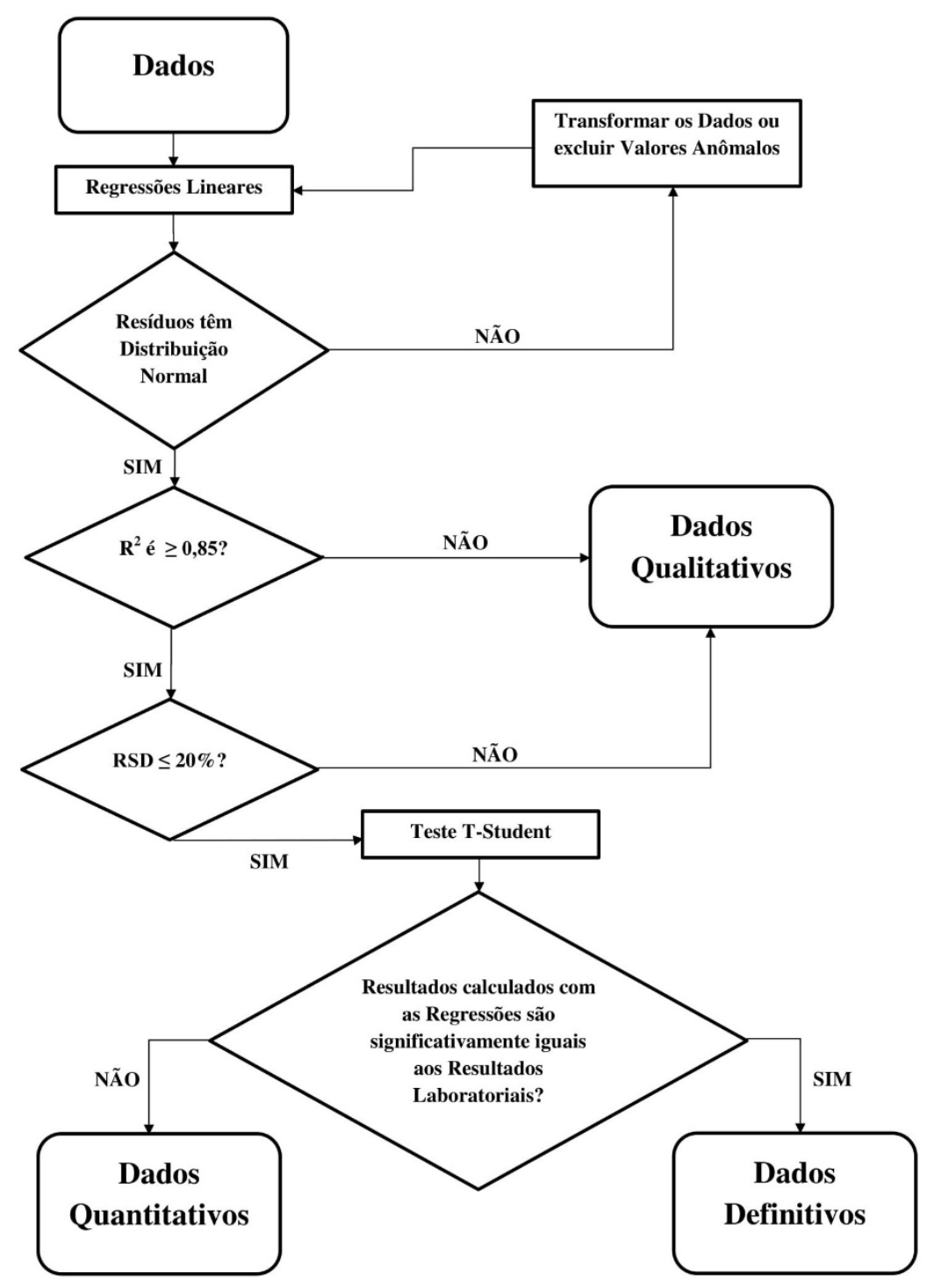

Figura. 2 - Fluxograma da Sequência das Análises Estatísticas (Adaptado de Shuttleworth et al., 2014). 


\section{RESULTADOS E DISCUSSÃO}

$\mathrm{Na}$ área investigada no presente estudo, dentre os elementos analisados com equipamento XRF$\mathrm{P}$, apenas o chumbo apresentou concentrações superiores aos valores de intervenção adotados à época pelo órgão ambiental, para ambientes agrícolas na matriz solo $(180 \mathrm{mg} / \mathrm{kg})$. Dos 705 pontos investigados, apenas 49 apresentaram valores acima de $180 \mathrm{mg} / \mathrm{kg}$ na profundidade mais rasa $(0,0$ a $0,1 \mathrm{~m}), 24$ na profundidade intermediária $(0,1$ a $0,2 \mathrm{~m})$ e 2 na maior profundidade $(0,2$ a $0,3 \mathrm{~m})$. A figura 3 ilustra a distribuição espacial das concentrações de chumbo para a camada de solo entre 0,0 e $0,1 \mathrm{~m}$ de profundidade.

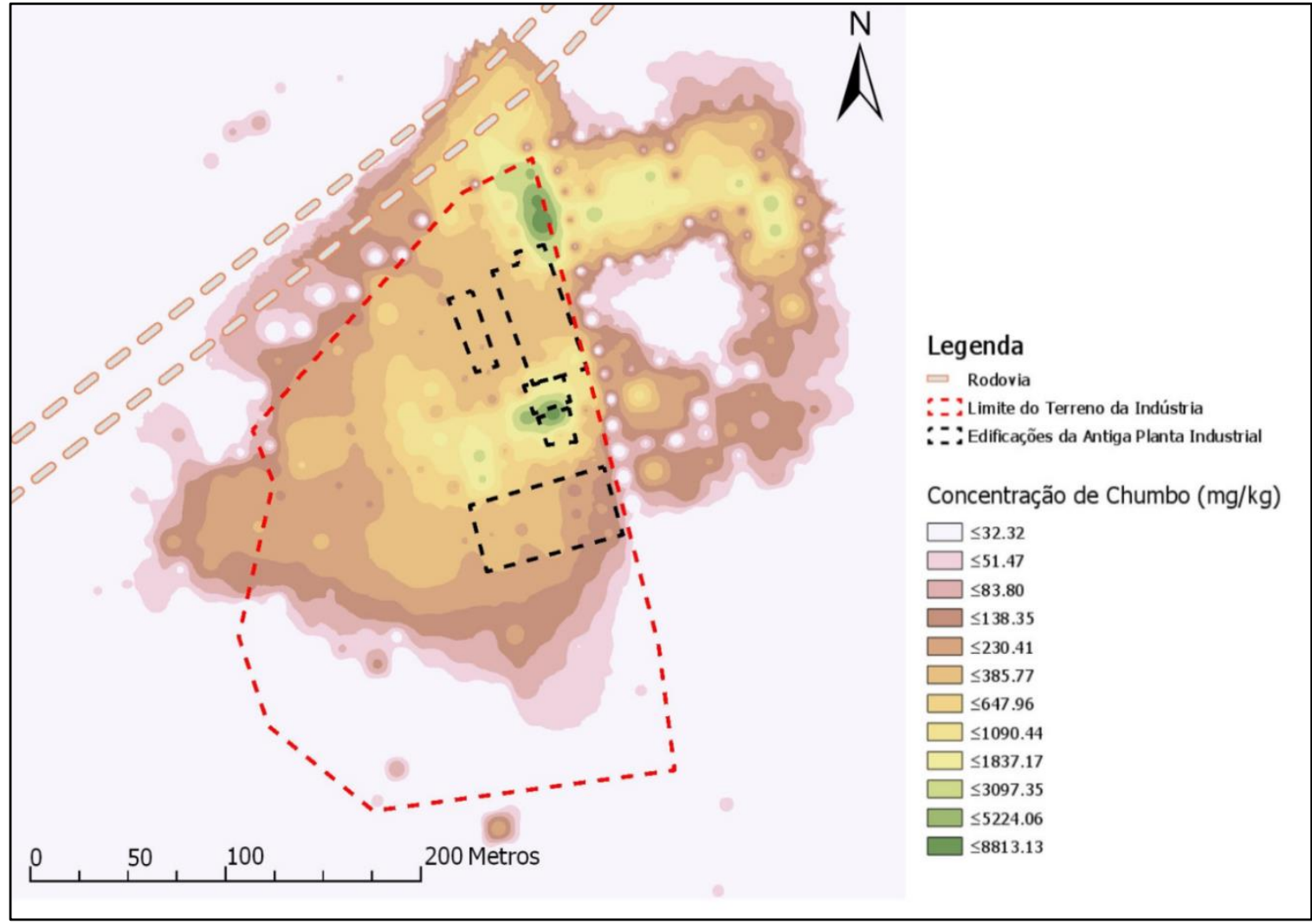

Figura 3 - Distribuição espacial das concentrações de chumbo no solo entre 0 - 0,1 m de profundidade.

Para verificação da qualidade dos dados obtidos com XRF-P realizou-se a correlação linear entre os resultados obtidos pelos dois métodos, que é apresentada na figura 4 (a) e apresenta coeficientes de determinação $\left(\mathrm{R}^{2}\right)$ de 0,97 . Apesar de o $\mathrm{R}^{2}$ estar próximo a 1 , a análise dos resíduos, apresentados na figura 4 (b), revela que eles são elevados, variando de 715 a $1539 \mathrm{mg} / \mathrm{kg}$ e até concentrações entorno de $2.600 \mathrm{mg} / \mathrm{kg}$ há um padrão exponencial na sua distribuição. Desta concentração em diante parece não haver padrão nos resíduos. Isto indica que a regressão não é adequada, apesar de apresentar $\mathrm{R}^{2}$ elevado, e que os dados devem ser transformados.

Realizou-se a transformação logarítmica dos dados, como recomendado por USEPA (1998) para comparação dos resultados obtidos para metais por XRF-P e ensaios laboratoriais, e foram ajustados modelos de regressão linear aos dados transformados, conforme a equação 3 :

$$
\log Y=m \times(\log X)+c \text {. (Equação } 3)
$$

Onde, Y são valores relativos aos ensaios laboratoriais (variável dependente), X a concentração de $\mathrm{Pb}$ obtida pelo equipamento de XRF-P (variável independente), c a intersecção da reta no eixo y e $m$ é a inclinação da reta de regressão linear para os dados transformados.

As figuras 5 e 6 ilustram estas regressões e seus resíduos, considerando-se o parâmetro c igual a zero e diferente de 0. Observaram-se coeficientes de determinação novamente próximos a 1 , tanto para a regressão linear com $c \neq 0 \quad\left(R^{2}=0,98\right)$, quanto para a regressão linear com $c=0\left(R^{2}=0,97\right)$. Verifica-se que em ambos os casos os resíduos distribuem-se em torno de zero, portanto a regressão com os dados transformados em escala logarítmica de base 10 é aceitável. 


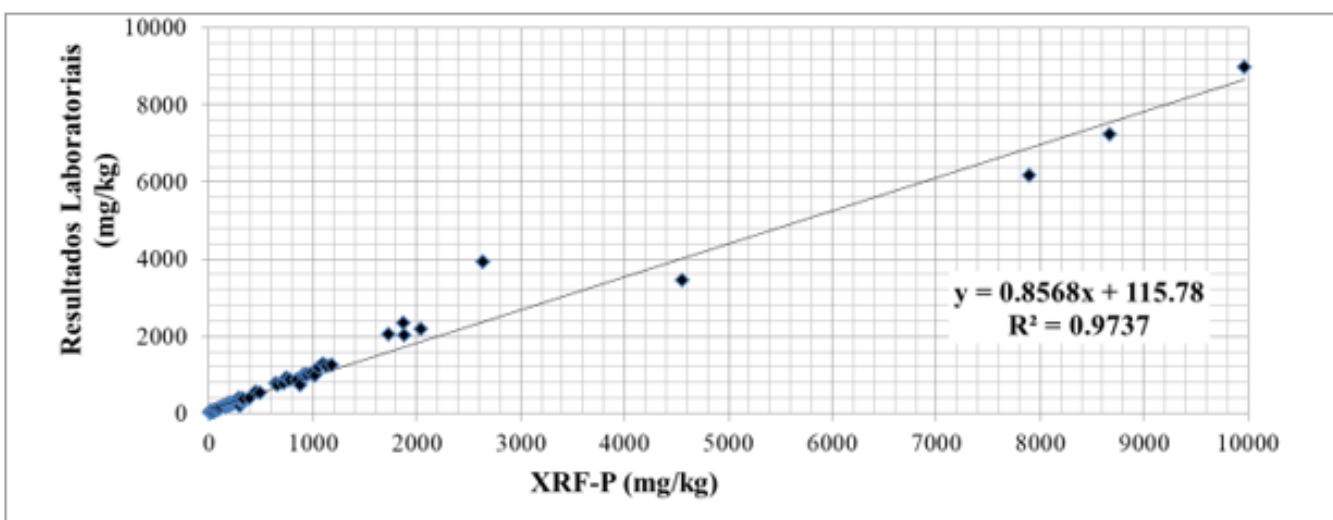

(a)

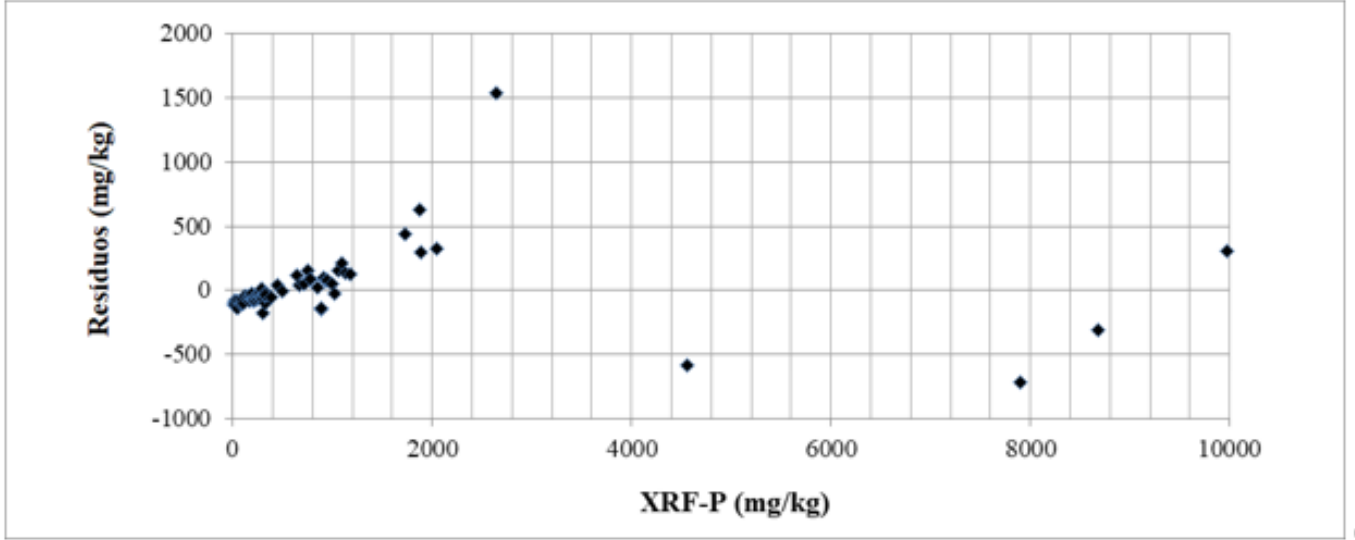

(b)

Figura 4 - (a) Regressão linear entre dados laboratoriais e os dados obtidos com XRF-P para todas as amostras que apresentaram resultados acima do limite de detecção do XRF-P (considerando cł0); (b) distribuição dos resíduos.
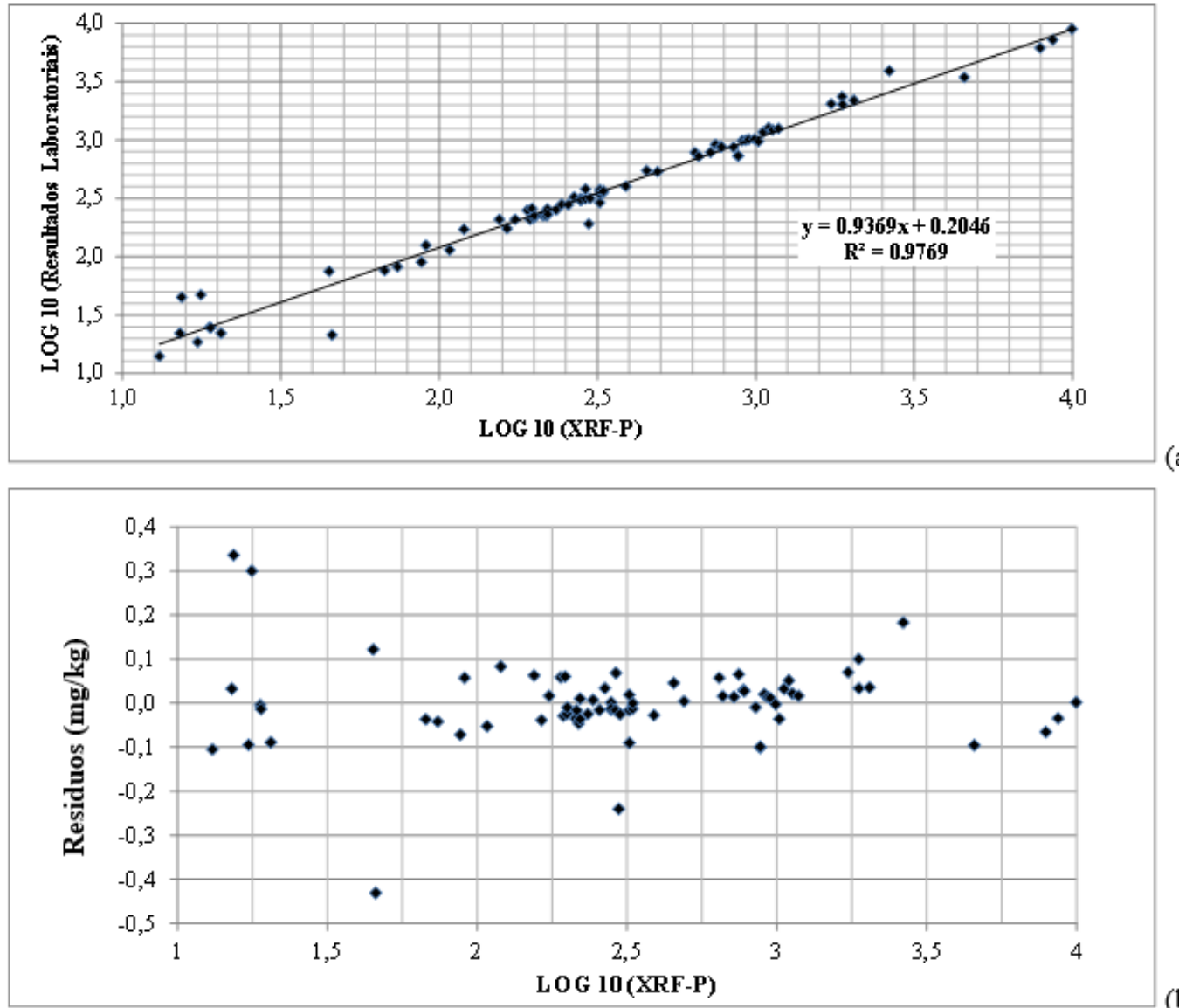

Figura 5 - (a) Regressão linear entre Log dos dados Laboratoriais e log dos dados obtidos pelo XRF-P (considerando $c \neq 0$ ), com todas as amostras que apresentaram resultados acima do limite de detecção do XRF-P; (b) distribuição dos resíduos. 
A próxima verificação a ser realizada diz respeito à precisão dos equipamentos. Neste estudo foi realizada apenas uma determinação para cada amostra, o que impede o cálculo do $\mathrm{RSD}$, entretanto a literatura técnica sobre testes em equipamentos XRF-P revela que a precisão destes equipamentos é bastante satisfatória, normalmente indicando valores abaixo de $8,8 \%$ para o elemento chumbo (Kilbride, 2006, Shuttleworth et al., 2014, Rouillon \& Taylor, 2016). Consideramos assim, que para o caso em questão, o RSD provavelmente é inferior a $20 \%$ e pode-se afirmar que o XRF-P fornece dados precisos.

Assim sendo, com base no critério de avaliação da qualidade dos dados proposto por USEPA (1998) os resultados obtidos pelo equipamento XRF-P neste estudo poderiam ser considerados definitivos e a regressão linear poderia ser aplicada sobre os resultados transformados na escala logarítmica de base 10 para a obtenção de concentrações de chumbo precisas e acuradas segundo este critério.

Para a aplicação da proposta de Shuttleworth et al. (2014), mais rigorosa que a de USEPA (1988), foi realizado o teste t-pareado, comparando-se os logaritmos dos valores de concentração de chumbo calculados pela regressão com os logaritmos dos valores de concentração de chumbo obtidos por ensaios laboratoriais. A figura 7 apresenta os histogramas dos dois conjuntos de dados. Verificase que, apesar de as distribuições serem semelhantes, o p-valor calculado por este teste vale 0,2961. Ele se localiza fora do intervalo de $95 \%$, ou seja, a hipótese nula deve ser rejeitada em favor da hipótese alternativa e as amostras não podem ser consideradas similares entre si no nível de significância de 95\%. Desta forma, pelo critério proposto por Shuttleworth et al. (2014) os dados obtidos com o XRF-P não podem ser considerados definitivos, mas podem ser considerados quantitativos, como exposto anteriormente. Assim sendo, a correlação apresentada na Equação 4 fornece valores quantitativos para cálculo das concentrações de chumbo no solo no caso em questão

\section{$\log Y=1,0137(\log X)($ Equação 4)}

Onde Y são os valores de concentração de chumbo estimados $(\mathrm{mg} / \mathrm{kg})$ e $\mathrm{X}$ os dados obtidos via XRF-P (mg/kg).
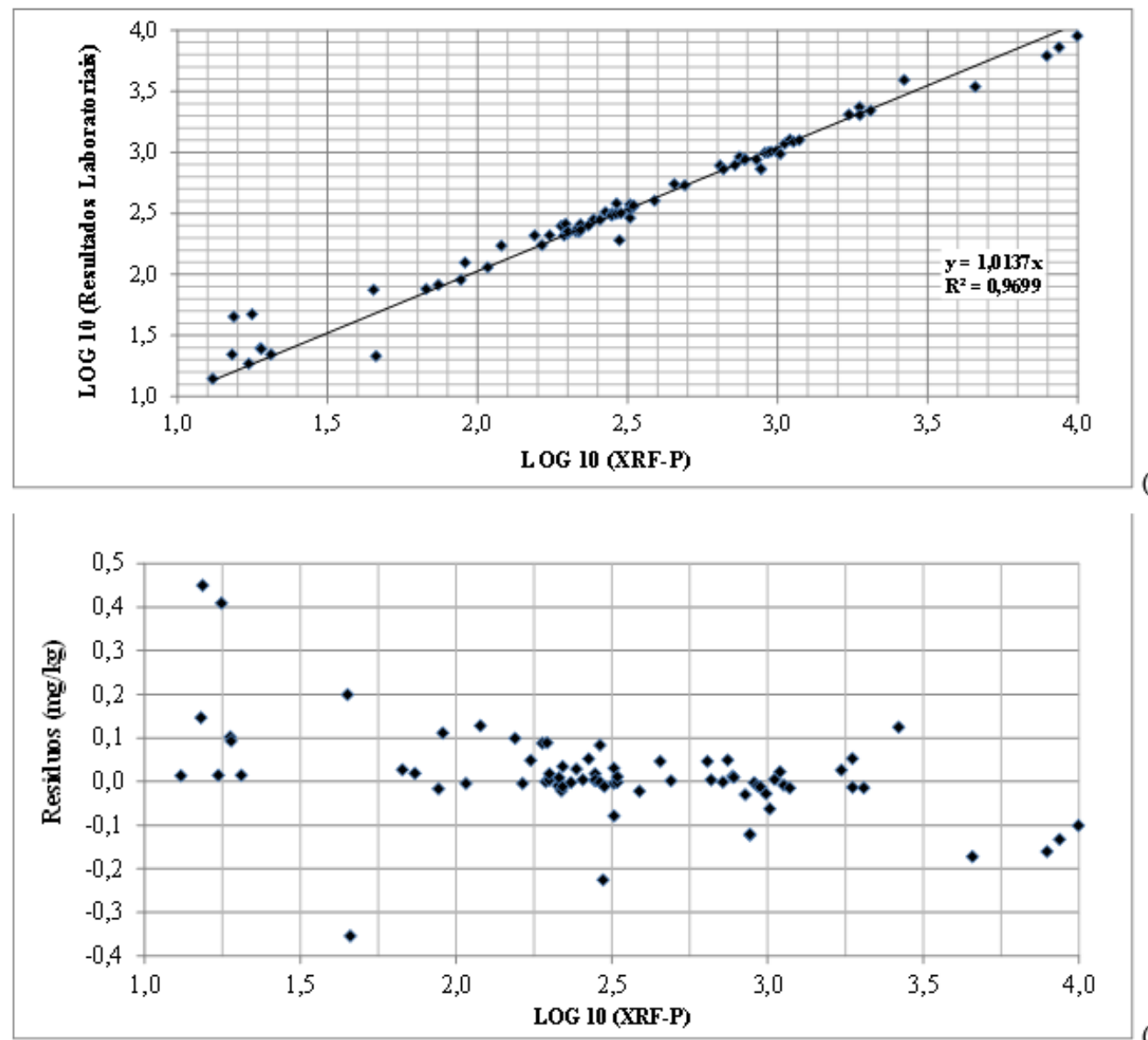

(b)

Figura 6 - (a) Regressão linear entre Log dos dados Laboratoriais e Log dos dados obtidos pelo XRF-P (considerando $\mathrm{c}=0$ ), com todas as amostras que apresentaram resultados acima do limite de detecção do XRF-P; (b) distribuição dos resíduos. 


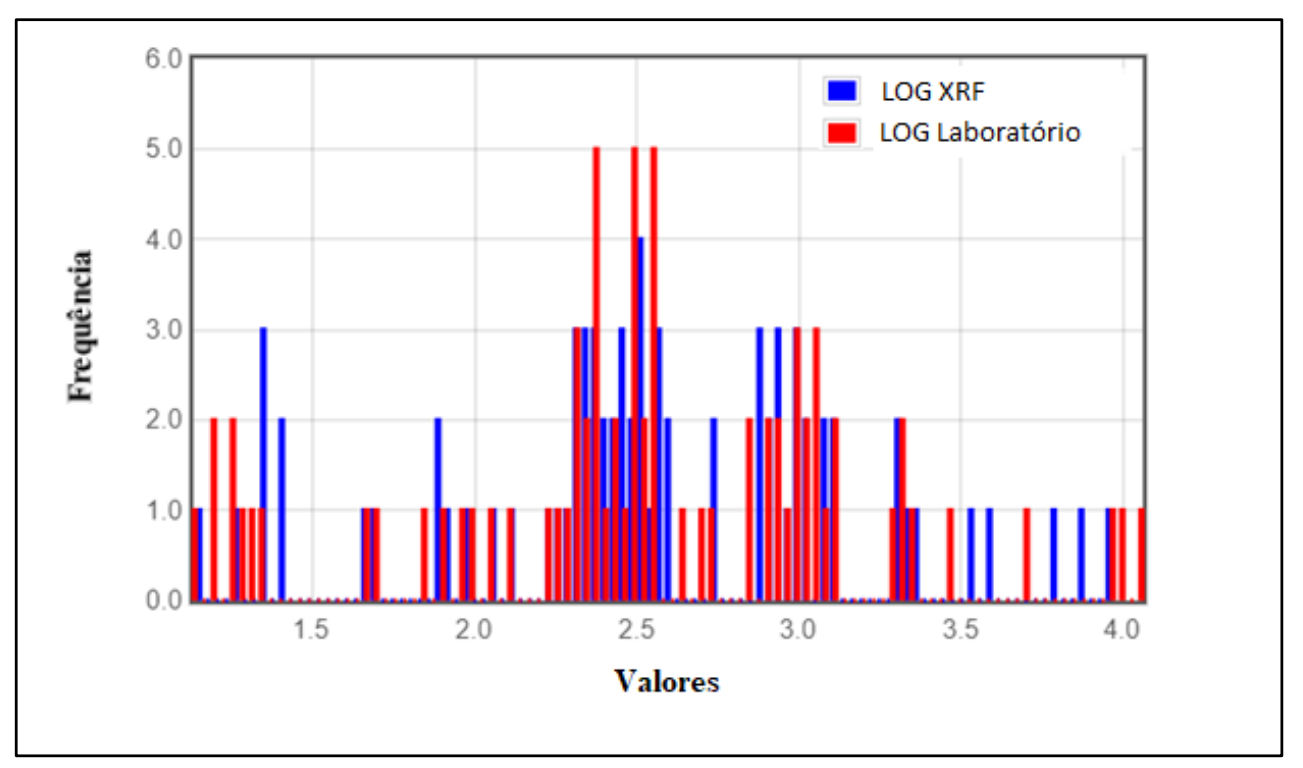

Figura 7 - Histograma dos Grupos de Dados utilizados no Teste T-pareado; Logaritmo das Concentrações de Chumbo Estimadas por Regressão Linear, identificados como "LOG XRF" e Logaritmo das Concentrações de Chumbo determinadas nos Ensaios Laboratoriais para todas as concentrações acima do limite de detecção do XRF.

Em vista do comportamento diferenciado apresentado pelos resíduos das concentrações superiores a $2.000 \mathrm{mg} / \mathrm{kg}$ em todas as correlações calculadas anteriormente, procedeu-se também à análise dos dados apenas para concentrações de chumbo no solo, inferiores a 10 vezes o valor de intervenção recomendado para áreas agrícolas $(180 \mathrm{mg} / \mathrm{kg})$. Este universo corresponde a 66 pares de dados, para os quais se realizou a transformação logarítmica e a verificação dos resíduos e dos $\mathrm{R}^{2}$ das regressões lineares e o teste t-pareado, conforme fluxograma da figura 2 .

A figura 8 (a) apresenta a regressão linear, considerando $\mathrm{c}=0$ e a figura 8 (b) mostra a distribuição de seus resíduos. A Figura 9 apresenta os histogramas dos dois conjuntos de dados utilizados neste teste de hipótese. Verificase que, apesar de as distribuições serem semelhantes, o p-valor calculado por este teste vale 0,4138 . Assim sendo, os valores estimados pela regressão linear considerando-se apenas concentrações inferiores a $1.800 \mathrm{mg} / \mathrm{kg}$ não são similares aos valores determinados em laboratório.

Comparando-se o valor de $\mathrm{R}^{2}$ para esta regressão com o valor obtido para a regressão realizada com os logaritmos das concentrações de todos os ensaios verifica-se que $\mathrm{R}^{2}$ do segundo caso é maior. Comparando-se o p-valor de ambos os testes t-pareado, verifica-se que ambos resultaram na rejeição da hipótese nula em favor da hipótese alternativa, porém p-valor é menor para a regressão que considera todos os resultados de ensaios. Desta forma, recomendase a adoção da Equação 4 para estimativa das concentrações de $\mathrm{Pb}$ no solo na área em questão a partir dos resultados dos ensaios realizados com XRF-P.

\section{CONCLUSÃO}

Setenta e cinco resultados de ensaios pareados de determinação da concentração de chumbo na matriz solo realizados em campo com equipamento XRF-P e em laboratório com procedimentos normatizados, em amostras coletadas em uma área sob investigação ambiental, foram tratados com técnicas estatísticas de regressão linear e teste de hipóteses.

As regressões lineares entre os resultados de ambos os ensaios exibiram sistematicamente coeficiente de determinação $\left(\mathrm{R}^{2}\right)$ próximo a 1 . A análise dos resíduos destas regressões revelou que elas são adequadas quando se aplicam aos dados uma transformação do tipo log-log. Os testes t-pareado realizados revelaram, entretanto, que as concentrações calculadas com a equação de regressão não podem ser consideradas idênticas às concentrações determinadas em laboratório, pois os $\mathrm{p}$-valores foram sempre superiores a 0,05 .

Para o caso em estudo, foi possível desenvolver uma equação de regressão linear para estimativa dos valores de concentração de chumbo a partir dos resultados obtidos com o equipamento XRF-P transformados (logaritmo de base 10). 
Considerando-se que os aparelhos XRF-P são instrumentos precisos, pois exibem normalmente RSD inferior a $20 \%$, pode-se classificar a qualidade dos dados obtidos com XRF-P como definitiva segundo USEPA (1998) e quantitativa segundo Shuttleworth et al. (2014).

Ressalta-se que a equação de correlação desenvolvida é específica para o caso em questão, porém o procedimento de tratamento estatístico dos dados pode ser repetido em outros casos para os quais se disponham de análises pareadas, de tal forma que o XRF-P possa ser utilizado como um instrumento para estimativa de resultados quantitativos, em oposição à prática corrente de empregá-lo apenas como investigação qualitativa.

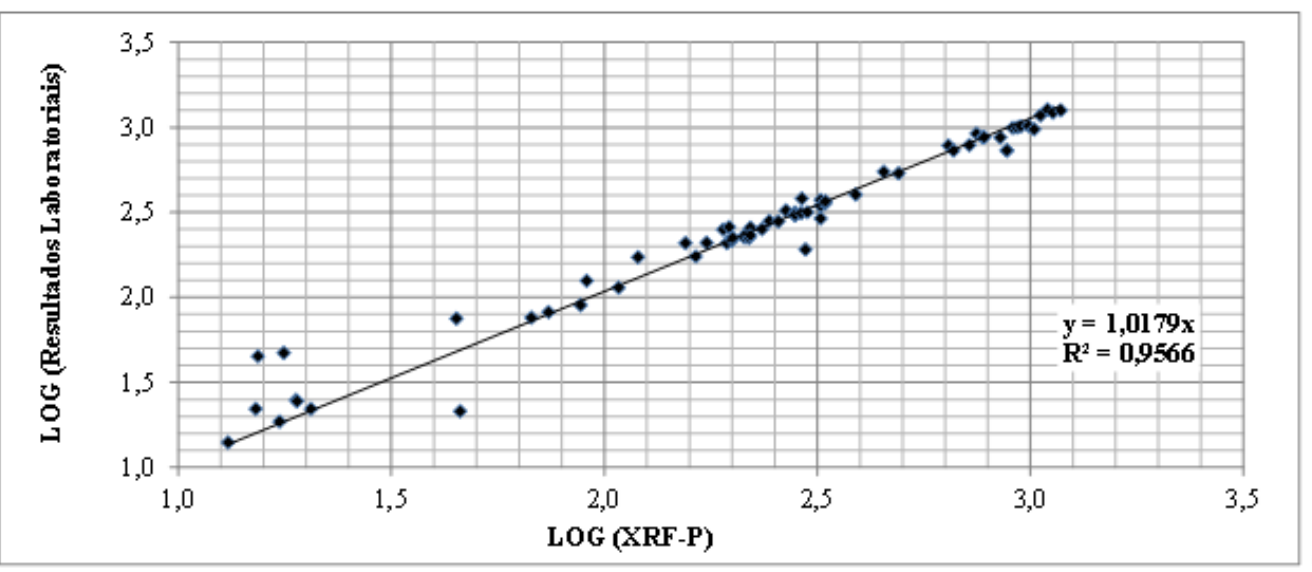

(a)

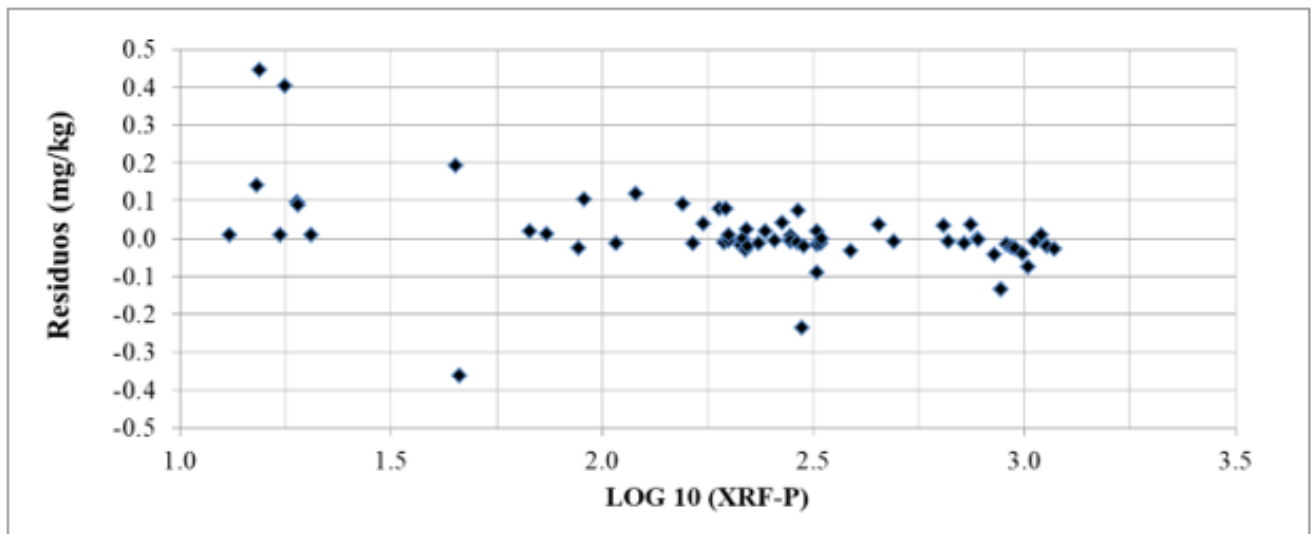

(b)

Figura 8 - Regressão linear entre Log dos dados Laboratoriais e Log dos dados obtidos pelo XRF-P, para valores abaixo de $1800 \mathrm{mg} / \mathrm{kg}$ (considerando c=0); (b) distribuição dos resíduos.

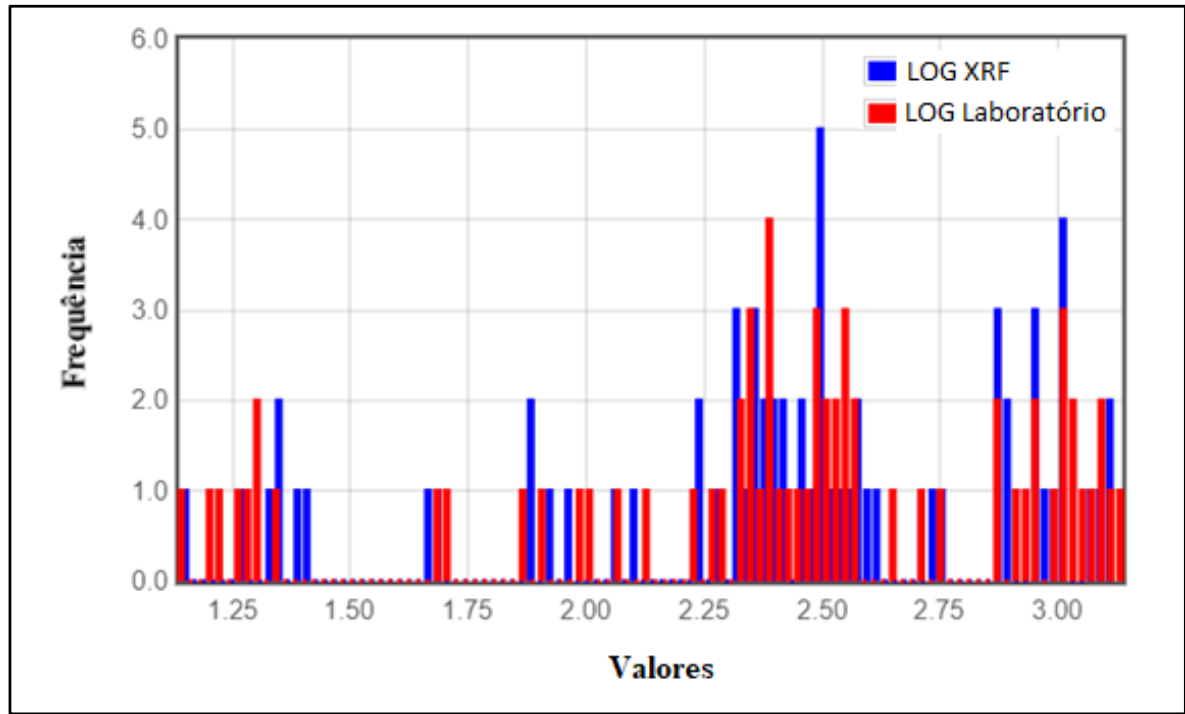

Figura 9 - Histograma dos Grupos de Dados utilizados no Teste T-pareado: Logaritmo das Concentrações de Chumbo Estimadas por Regressão Linear, identificados como "LOG XRF" e Logaritmo das Concentrações de Chumbo determinadas nos Ensaios Laboratoriais, para valores abaixo de $1800 \mathrm{mg} / \mathrm{kg}$. 


\section{AGRADECIMENTOS}

Os autores agradecem os proprietários da área e a empresa de consultoria envolvida no caso em questão pelo fornecimento dos dados e pela autorização para publicação deste artigo.

\section{REFERÊNCIAS}

ALLOWAY, B.J. Heavy Metals in Soils: Sources of Heavy Metals and Metalloids in Soils. Environmental Pollution, v. 22, p.11-50, 2013.

AYDINALP, C. \& MARINOVA, S. The Effects of Heavy Metals on seed germination and plant growth on aldalfa plant (MEDICAGO SATIVA). Bulgarian Journal of Agricultural Science, v. 15, p. 347- 350, 2009.

CETESB - COMPANHIA DE TECNOLOGIA DE SANEAMENTO AMBIENTAL. Decisão de Diretoria $\mathbf{N}^{\circ}$ 195-2005- E. Valores Orientadores para Solos e Águas Subterrâneas no Estado de São Paulo. 2005

CETESB - COMPANHIA DE TECNOLOGIA DE SANEAMENTO AMBIENTAL. Decisão de Diretoria $\mathbf{N}^{\circ}$ 256-2016- E. Valores Orientadores para Solos e Águas Subterrâneas no Estado de São Paulo - 2016. 2016.

CETESB - COMPANHIA DE TECNOLOGIA DE SANEAMENTO AMBIENTAL. Manual de Gerenciamento de Áreas Contaminadas. São Paulo, SP, 1999.

DAVIDSON, C.M. Heavy Metals in Soils: Methods for the Determination of Heavy Metals and Metalloids in Soils. Environmental Pollution. v. 22, p. 97-140, 2013.

DISCENZA, D.J.; KEIMOWITZ, A.R.; FITZGERALD, N. Calibration and Evaluation of an X-Ray Fluorescence Method for the Determination of Lead and Arsenic in Soils. Journal of Environmental Analytical Chemistry, v. 01, p.1-3, 2014.

GUILHERME, L.R.G.; MARQUES, J.J.; PIERANGELI, M.A.P.; ZULIANI, D.Q.; CAMPOS, M.L.; MARCHI, G. Elementos-traço em solos e sistemas aquáticos. Tópicos em Ciência do Solo, v. 4, p. 345 - 390, 2005.

HU, B.; CHEN, S.; HU, J.; XIA, F. Application of portable XRF and VNIR sensors for rapid assessment of soil heavy metal pollution. Plos One, v. 12, p.1-13, 2017.

KILBRIDE, C.; POOLE, J.; HUTCHINGS, T.R. A comparison of $\mathrm{Cu}, \mathrm{Pb}, \mathrm{As}, \mathrm{Cd}, \mathrm{Zn}, \mathrm{Fe}, \mathrm{Ni}$ and $\mathrm{Mn}$ determined by acid extraction/ICP-OES and ex situ field portable X-ray fluorescence analyses. Environmental Pollution, v. 143, p.1623,2006
MANAHAN, S.E. Environmental Science Technology and Chemistry, Boca Raton: CRC Press LLC, 743 p. 2000.

ROUILLON, M \& TAYLOR, M. P. Can field portable X-ray fluorescence (pXRF) produce high quality data for application in environmental contamination research? Environmental Pollution, v. 214, p.255-264, 2016.

SHUTTLEWORTH, E.L.; EVANS, M.G.; HUTCHINSON. S.M.; ROTHWELL, J.J. Assessment of Lead Contamination in Peatlands Using Field Portable XRF. Water, Air, \& Soil Pollution, v. 225, p.1-13, 2014.

SPARKS, D.L. Environmental Soil Chemistry. San Diego: Academic Press, 267 p. 1995.

USEPA - United States Environmental Protection Agency. Method 3050B: Acid Digestion of Sediments, Sludges, and Soils. US EPA, 1996.

USEPA - United States Environmental Protection Agency. Method 6010B: Inductively Coupled Plasma-Atomic Emission Spectrometry. US EPA, 1998.

USEPA - United States Environmental Protection Agency. Environmental Technology Verification Report: Field Portable X-Ray Fluorescence Analyzer. US EPA, 2007.

USEPA - United States Environmental Protection Agency. Method 6200: Field Portable X-Ray Fluorescence Spectrometry for the Determination of Elemental Concentrations in Soil and Sediment. US EPA, 2007.

WONG, C.; XIANG-DONG, L.; THORNTON, I. Urban environmental geochemistry of trace metals. Environmental Pollution, v. 142, p. 1-16, 2006

Submetido em 28 de novembro de 2017 Aceito para publicação em 11 de junho de 2020 